TITLE:

\title{
Visualization of carotid vessel wall and atherosclerotic plaque: T-SPACE vs. compressed sensing T-SPACE
}

\section{$\operatorname{AUTHOR}(S)$ :}

Okuchi, Sachi; Fushimi, Yasutaka; Okada, Tomohisa; Yamamoto, Akira; Okada, Tsutomu; Kikuchi, Takayuki; Yoshida, Kazumichi; Miyamoto, Susumu; Togashi, Kaori

\section{CITATION:}

Okuchi, Sachi ... [et al]. Visualization of carotid vessel wall and atherosclerotic plaque: T-SPACE vs. compressed sensing T-SPACE. European Radiology 2019, 29(8): 4114-4122

\section{ISSUE DATE:}

2019-08

URL:

http://hdl.handle.net/2433/242961

\section{RIGHT:}

This is a post-peer-review, pre-copyedit version of an article published in 'European Radiology'. The final authenticated version is available online at: https://doi.org/10.1007/s00330-018-5862-8; The full-text file will be made open to the public on 06 December 2019 in accordance with publisher's 'Terms and Conditions for Self-Archiving'; この論文は出版 社版でありません。引用の際には出版社版をご確認ご利用ください。; This is not the published version. Please cite only the published version. 


\section{Title}

Visualization of Carotid Vessel Wall and Atherosclerotic Plaque: T1-SPACE vs.

Compressed Sensing T1-SPACE

Sachi Okuchi, $\mathrm{MD}, \mathrm{PhD}^{1}$, Yasutaka Fushimi, $\mathrm{MD}, \mathrm{PhD}^{1}$, Tomohisa Okada, $\mathrm{MD}, \mathrm{PhD}^{2}$, Akira Yamamoto, $\mathrm{MD}, \mathrm{PhD}^{1}$, Tsutomu Okada, $\mathrm{MD}, \mathrm{PhD}^{1}$, Takayuki Kikuchi, $\mathrm{MD}$, $\mathrm{PhD}^{3}$, Kazumichi Yoshida, $\mathrm{MD}, \mathrm{PhD}^{3}$, Susumu Miyamoto, $\mathrm{MD}, \mathrm{PhD}^{3}$, and Kaori Togashi, MD, $\mathrm{PhD}^{1}$

${ }^{1}$ Department of Diagnostic Imaging and Nuclear Medicine, Kyoto University Graduate School of Medicine, Kyoto, 606-8507 JAPAN.

${ }^{2}$ Human Brain Research Center, Kyoto University Graduate School of Medicine, Kyoto, 606-8507 JAPAN.

${ }^{3}$ Department of Neurosurgery, Kyoto University Graduate School of Medicine, Kyoto, 606-8507 JAPAN.

\section{*Corresponding Author: Yasutaka Fushimi, MD, PhD}

Department of Diagnostic Imaging and Nuclear Medicine, Kyoto University Graduate School of Medicine, 54 Shogoin Kawahara-cho, Sakyo-ku, Kyoto, 606-8507, Japan

Phone: +81-75-751-3760, FAX: +81-75-771-9709

E-mail: yfushimi@kuhp.kyoto-u.ac.jp

1905 words

\section{Financial disclosures}

None.

\section{Funding information}

This work was partly supported by Grant-in-Aid for Scientific Research on Innovative Areas "Initiative for High-Dimensional Data-Driven Science through Deepening of Sparse Modeling”, Ministry of Education, Culture, Sports, Science and Technology (MEXT) grant numbers 25120002, 25120008, and supported by The Kyoto University Foundation. 


\section{Acknowledgement}

We are grateful to Mr. Katsutoshi Murata and Mr. Yuta Urushibata, Siemens Healthcare Japan K. K., for protocol optimization. This study was approved by Ethics Committee Graduate School and Faculty of Medicine Kyoto University.

\section{E-mail}

sachi.okuchi@gmail.com

yfushimi@kuhp.kyoto-u.ac.jp

tomokada@kuhp.kyoto-u.ac.jp

yakira@kuhp.kyoto-u.ac.jp

tokadat@kuhp.kyoto-u.ac.jp

tkik@kuhp.kyoto-u.ac.jp

kazuy@kuhp.kyoto-u.ac.jp

miy@kuhp.kyoto-u.ac.jp

ktogashi@kuhp.kyoto-u.ac.jp 
Title of the manuscript: Visualization of Carotid Vessel Wall and Atherosclerotic Plaque: T1SPACE vs. Compressed Sensing T1-SPACE

Article Type: Original Article

Key Points

1. CS-T1-SPACE allowed better visualization compared with T1-SPACE in evaluating carotid plaques and vessel walls with a 2.5-fold accelerated scan time with comparable image quality.

2. CS-T1-SPACE offers a promising method for investigating carotid vessel walls due to the better image quality with shorter acquisition time.

3. Physiological movements such as swallowing, arterial pulsations, and breathing induce motion artifacts in vessel wall imaging, and a shorter acquisition time can reduce artifacts from physiological movements.

Keywords: Magnetic Resonance Imaging; Carotid Stenosis; Atherosclerosis; Artifacts; Image Reconstruction

List of all abbreviations with spelled out words:

T1-SPACE, T1-fast spin echo imaging with conventional SPACE;

CS, compressed sensing;

$\mathrm{SR}$, signal intensity ratio;

VWI, vessel wall imaging;

FSE, fast spin echo;

ICA, internal carotid artery;

mFISTA, Modified Fast Iterative Shrinkage-Thresholding Algorithm;

PI, parallel imaging;

g-factor, geometry factor 


\section{Abstract}

\section{Objectives}

To compare visualization of carotid plaques and vessel walls between 3D T1-fast spin echo imaging with conventional SPACE (T1-SPACE) and with a prototype compressed sensing T1-SPACE (CS-T1SPACE).

\section{Methods}

This retrospective study was approved by the institutional review board. Participants comprised 43 patients (36 males, 7 females; mean age, 71 years) who underwent carotid MRI including T1-SPACE and CS-T1-SPACE. Quality of visualization for carotid plaques and vessel walls was evaluated using a 5-point scale, and signal intensity ratios (SRs) of the carotid plaques were measured and normalized to adjacent sternomastoid muscle. Scores for the quality of visualization were compared between T1SPACE and CS-T1-SPACE using the Wilcoxon signed-rank test. Statistical differences between SRs of plaques with T1-SPACE and CS-T1-SPACE were also evaluated using the Wilcoxon signed-rank test, and Spearman’s correlation coefficient was calculated to investigate correlations.

\section{Results}

Visualization scores were significantly higher for CS-T1-SPACE than for T1-SPACE when evaluating carotid plaques $(P=0.0212)$ and vessel walls $(P<0.001)$. The SR of plaques did not differ significantly between T1-SPACE and CS-T1-SPACE $(P=0.5971)$. Spearman's correlation coefficient was significant $(0.884 ; P<0.0001)$. 


\section{Conclusions}

CS-T1-SPACE allowed better visualization scores and sharpness compared with T1-SPACE in evaluating carotid plaques and vessel walls with a 2.5-fold accelerated scan time with comparable image quality. CS-T1-SPACE appears promising as a method for investigating carotid vessel walls, offering better image quality with a shorter acquisition time. 
Visualization of Carotid Vessel Wall and Atherosclerotic Plaque: T1-SPACE vs. Compressed

\section{Sensing T1-SPACE}

\section{Introduction}

Stroke is the second leading cause of death worldwide [1]. Intra-plaque hemorrhage is a critical factor in the growth and destabilization of atherosclerotic plaques [2], and rupture of carotid atherosclerotic plaque accounts for around $20 \%$ of the causes of all ischemic strokes [3]. Atherosclerotic change is also associated with silent infarcts and white matter disease [4]. Establishing methods for visualizing carotid plaque and determining whether a plaque contains substantial amounts of vulnerable components such as intraplaque hemorrhage is therefore important.

The characteristics of carotid plaque provided by plaque imaging on MR can serve a surrogate marker for risk management of patients with atherosclerosis [5]. The characteristics of carotid plaque such as intraplaque hemorrhage, calcification, and lipid-rich necrotic core are evaluated by threedimensional (3D) T1-weighted fast spin echo (T1-FSE) in combination with T2WI, time-of-flight MR angiography, magnetization-prepared rapid acquisition gradient echo sequences [6]. The 3D T1-FSE accurately characterizes intraplaque components of the carotid artery, with excellent sensitivity and specificity due to the higher spatial resolution compared with 2D-T1WI, because this sequence can minimize partial volume effects [7]. However, the long acquisition time represents a substantial drawback for 3D sequences [8]. Moreover, physiological movements such as swallowing, arterial 
pulsation, and breathing induce motion artifacts in carotid vessel wall imaging (VWI) [9].

T1-weighted SPACE (T1-SPACE) is a 3D FSE sequence with variable flip angle and has been used in VWI because of the high sampling efficiency and good blood suppression properties in clinical practice [8]. Recently, a compressed sensing (CS) algorithm has been introduced to MRI, including time-of-flight MR angiography [10; 11], MR-DSA [12] and dynamic contrast-enhanced MRI [13]. CS has also been applied to SPACE imaging $[14 ; 15]$ to achieve shorter acquisition times, which reconstructed iteratively from incoherently under-sampled data using a regularization term [16].

A small number of studies have featured CS in the evaluation of carotid VWI [17-20], but the numbers of subjects in those preliminary papers were around 10 patients, and results were not conclusive. The purpose of this study was to compare the visualization of plaques and vessel walls of the internal carotid artery (ICA) between 3D T1-FSE imaging with conventional SPACE and with a prototype CS-SPACE in a larger number of cases.

\section{Materials and Methods}

This retrospective study was approved by the local institutional review board. The need to obtain informed consent was waived due to retrospective nature of the study.

\section{Subjects}

Forty-three consecutive patients (36 males, 7 females; mean age, 71 years; range, 25-86 years) who had undergone carotid MRI including 3D T1-FSE imaging with conventional SPACE (T1- 
SPACE) and with a prototype 3D CS-SPACE (CS-T1-SPACE) for assessing stenosis and plaque in the cervical ICA between June 2016 and February 2017 were evaluated in this study. The subjects were included if they met the following criteria: (a) patients who was suspected to have carotid stenosis or plaque by carotid ultrasound or clinical history such as recent cerebral infarction; (b) patients who underwent both T1-SPACE and CS-T1-SPACE. The subjects were excluded if they met the following exclusion criteria: patients who underwent either T1-SPACE or CS-T1-SPACE.

\section{Image Acquisition}

All scans were conducted using a 3-T MR system (MAGNETOM Skyra; Siemens Healthineers) with a 20-channel head coil.

Pulse sequence parameters were as follows. Conventional 3D T1-weighted SPACE (T1SPACE): coronal acquisition; TR/TE, 727/11.0 ms; variable flip angle; echo-train length, 32; FOV, $216 \mathrm{~mm} \times 240 \mathrm{~mm}$; voxel size, $0.75 \mathrm{~mm} \times 0.75 \mathrm{~mm} \times 0.75 \mathrm{~mm}$; GRAPPA, 3×; fat suppression, SPAIR; and acquisition time, 7 min 9 s. Prototype 3D CS-T1-SPACE: coronal acquisition; TR/TE, 650/11.0 ms; variable flip angle; echo-train length, 32; FOV, $240 \mathrm{~mm} \times 240 \mathrm{~mm}$, voxel size, 0.75 $\mathrm{mm} \times 0.75 \mathrm{~mm} \times 0.75 \mathrm{~mm}$, acceleration factor, 10×; fat suppression, SPAIR; and acquisition time, 2 $\min 49 \mathrm{~s}$.

CS-T1-SPACE was performed with an incoherent k-space undersampling pattern [21-24] using a Poisson-disc variable-density pattern combined with elliptical scanning and a fully sampled k- 
space center to allow for computation of coil sensitivities for image reconstruction [14].

CS-T1-SPACE data was seamlessly reconstructed on a standard reconstruction system, using a non-linear iterative SENSE-based algorithm with a constraint to enforce sparsity by solving the following minimization problem with a Modified Fast Iterative Shrinkage-Thresholding Algorithm (mFISTA).

$$
\min _{x}\left\{\frac{1}{2} \sum_{j=1}^{N}\left\|y_{j}-F_{u} S_{j} x\right\|_{2}^{2}+\lambda\|W x\|_{1}\right\}
$$

where $x$ is the image to reconstruct, $y_{j}$ and $S_{j}$ are the k-space data and coil sensitivity for the $j$-th coil element, $N$ is the number of coil elements, $F_{u}$ is the Fourier undersample operator, $W$ is the Haar wavelet transform, and $\lambda$ is the normalized regularization weighting factor. The first term is a data fidelity term, which ensures consistency between measured k-space data and the image estimate. The second term enforces sparsity and serves as a regularization term. Sparsity is promoted by minimizing the L1 norm in the wavelet domain, after applying the redundant Haar wavelet transform to the image of the current iteration. The regularization parameter $\lambda$ balances between sharpness, smoothness, noise and aliasing artifacts and a $\lambda$ of 0.001 was adopted [14]. Ten iteration times were adopted in this study to minimize reconstruction time while retaining adequate image quality. Total reconstruction was finished within $2 \min 15 \mathrm{~s}$.

To elucidate the effect of iterative reconstruction, CS-T1-SPACE images of one representative case were reconstructed with 1, 2, 3, 4, 5, 10, 15, 20, 25, 30, 35, 40, 45 and 50 iterations (Figure 1) to evaluate sharpness at the carotid artery edge for CS-T1-SPACE images with each iteration [25]. 
Sharpness was defined as the slope at the distance between $20 \%$ and $80 \%$ of maximum signal intensity for the Gaussian-fitted line profile of the carotid artery wall and the carotid lumen on the source image reconstructed with $1,2,3,4,5,10,15,20,25,30,35,40,45$ and 50 iterations, calculated using ImageJ software version 1.50 (http://imagej.nih.gov/ij/).

\section{Image Analysis}

The 3D image volumes of T1-SPACE and CS-T1-SPACE were registered to each other and reconstructed in axial images with a thickness of $0.75 \mathrm{~mm}$ (syngo Fusion; Siemens Healthineers). Bilateral plaques in the same subject were separately analyzed. The sequence name of each image was blinded. The quality of visualization for carotid plaques and vessel walls was evaluated using a 5-point scale (1, non-diagnostic; 2, poor; 3, acceptable; 4, good; 5, excellent). The score of the visualization of plaque and vessel wall were defined in Table 1. Evaluation was conducted by two radiologists independently (Y.F. with 20 years of experience; S.O. with 10 years of experience) using RadiAnt DICOM Viewer version 3.4.2 (Medixant).

To measure signal intensities, regions of interest (ROIs) were placed at the carotid plaques and adjacent sternomastoid muscle by the consensus decision of two radiologists (Y.F. and S.O.) using ImageJ software. When both raters graded plaque with score 2 or more, ROIs of the plaques were placed on the slice with maximal plaque size [7]. In case of small size of the plaque $(<3 \mathrm{~mm})$, measurement of plaque signal-intensity were not performed because the small plaque would have 
been biased due to the small ROI and associated partial volume effect. Plaque with black signals was also excluded. The signal intensity ratio (SR) was calculated by dividing the mean signal value of the plaque by the mean signal value of the sternomastoid muscle.

The plaque area size and plaque sharpness at the edge of the carotid plaque were also measured on the slice with maximal plaque using ImageJ software. Plaque size was manually measured to evaluate the correlation of the plaque size between T1-SPACE and CS-T1-SPACE. Sharpness of the plaque and vessel wall was defined as the slope between $20 \%$ and $80 \%$ of maximum signal intensity for the Gaussian-fitted line profile of the carotid plaque or the vessel wall and the carotid lumen.

\section{Statistical Analysis}

Agreement of independent measurements of visualization scores by two radiologists were evaluated using the inter-rater agreement statistic (kappa), and average scores from the two evaluators were used for statistical analyses.

Scores of the quality of visualization for carotid plaques and vessel walls were compared between T1-SPACE and CS-T1-SPACE using Wilcoxon signed-rank test because the data distribution was non-normal. The sharpness of plaque and vessel wall was also compared between T1-SPACE and CS-T1-SPACE using Wilcoxon signed-rank test. The significance of differences in SR of plaques between T1-SPACE and CS-T1-SPACE was also evaluated using the Wilcoxon signed-rank test, and Spearman's correlation coefficient was calculated to investigate correlations of 
SRs for plaques between T1-SPACE and CS-T1-SPACE. Spearman's correlation coefficient was also calculated to show the correlation of the plaque size between T1-SPACE and CS-T1-SPACE. Values of $P<0.05$ were considered statistically significant. Statistical analyses were conducted using commercially available MedCalc version 17.8.6 software.

\section{Results}

Edge sharpness of CS-T1-SPACE with 1, 2, 3, 4, 5, 10, 15, 20, 25, 30, 35, 40, 45 and 50 iterations and T1-SPACE is shown for a representative case (Figure 2). Edge sharpness rapidly increased from 1 to 10 iterations and became better than that with T1-SPACE. Edge sharpness slightly fluctuated between 10 and 50 iterations, and 10 iteration times were adopted. Representative clinical images are shown in Figures 3-5.

Carotid plaques were observed bilaterally in 32 patients and unilaterally in 6 patients and the number of plaques with visualization score $\geq 2$ were 63 for T1-SPACE, 70 for CS-T1-SPACE. The 63 identified plaques on T1-SPACE could be identified on CS-T1-SPACE. Seven plaques with visualization scores of 1 on T1-SPACE were excluded because definitive placement of ROIs was difficult, and the corresponding 7 plaques on CS-T1-SPACE were also excluded for the analysis. Five patients showed no plaques. Thirty-five plaques were excluded in the analysis of signal intensity, due to the small size of the plaque $(<3 \mathrm{~mm})$. Signal-intensity measurements performed on the small plaque would have been biased due to the small ROI and associated partial volume effect. We also excluded 
4 plaques with black signals. Finally, ROIs were drawn on 24 plaques on T1-SPACE and CS-T1SPACE (Supplementary Figure 1).

Kappa values of inter-rater agreement for the visualization of carotid plaques and vessel walls were 0.958 and 0.956 in T1-SPACE, confirming excellent agreement. Kappa values for carotid plaques and vessel walls were 0.713 and 0.657 in CS-T1-SPACE, showing good agreement.

Visualization scores were significantly higher in CS-T1-SPACE than in T1-SPACE for evaluating carotid plaques $(P=0.0212)$ and vessel walls $(P<0.001)$ (Figure 6).

Sharpness was measured for plaque and vessel wall in addition to visualization score. Plaques with visualization score $\geq 2$ were measured, namely, 63 for T1-SPACE, and corresponding 63 for CS-T1-SPACE and 7 patients with visualization scores of 1 on T1-SPACE were excluded. Sharpness of plaque was significantly better in CS-T1-SPACE (36.7: 95\% CI 31.4-41.5) than in T1-SPACE (30.8: 95\% CI 24.2-35.1) for evaluating carotid plaques $(P=0.0022)$. Sharpness of vessel wall was better in CS-T1-SPACE (54.8: 95\%CI 22.9-27.8) than in T1-SPACE (42.5: 95\% CI 21.4-25.5), however, with no statistical significance $(P=0.129)$.

SRs of plaques between T1-SPACE and CS-T1-SPACE showed no significant difference $(P=0.5971)$. Spearman's rank correlation coefficient (rho) for the SR between T1-SPACE and CS-T1SPACE was $0.884(P<0.0001)$, which was significant (Supplementary Figure 2).

The area of plaque was $30.5 \pm 19.2 \mathrm{~mm}^{2}$ for T1-SPACE and $31.2 \pm 19.7 \mathrm{~mm}^{2}$ for CS-T1-SPACE. Spearman's rank correlation coefficient (rho) for the plaque size between T1-SPACE and CS-T1- 
SPACE was $0.991 \quad(P<0.0001)$ (Supplementary Figure 3), therefore, there may exist some overestimation of plaque size on CS-T1-SPACE probably due to some blurring seen in CS-T1-SPACE.

\section{Discussion}

This study reported the successful clinical application of a CS technique to T1-SPACE in carotid vessel wall imaging. CS-T1-SPACE offered a higher quality of visualization of the carotid plaques and vessel walls than T1-SPACE with statistical significance. The number of recognized plaques were 63 for T1-SPACE, and 70 for CS-T1-SPACE, which represents 7 plaques were only identified on CST1-SPACE. It was hard recognized these 7 plaques on T1-SPACE probably due to artifacts, on the contrary, we were able to recognize them on CS-T1-SPACE due to less artifacts. In CS-T1-SPACE, no cases had visualization scores of 1 and only a few cases had scores of 2 probably because less artifact was seen during shorter scan time compared with T1-SPACE. CS-T1-SPACE provided significantly better plaque sharpness compared with T1-SPACE. Images of CS-T1-SPACE looked slightly blurred than T1-SPACE in most of the cases, but sharpness measured in CS-T1-SPACE was statistically better than that in T1-SPACE. Higher plaque sharpness was seen in CS-T1-SPACE than T1-SPACE, which suggested that the difference of signal intensity between plaque and intravascular lumen was larger in CS-T1-SPACE compared with T1-SPACE. The reason of higher sharpness in CST1-SPACE was probably due to the iterative reconstruction in CS which made the signal of intravascular lumen darker. As shown in Figure 2, sharpness of CS-T1-SPACE becomes larger 
according to the number of iteration times between 1 and 10 times. During multiple iterative reconstruction process, the intravascular faint signal intensity which is often seen in T1-SPACE were removed. Hence, the larger difference of signal intensity between plaque and intravascular lumen in CS-T1-SPACE resulted in better visualization score in CS-T1-SPACE compared with T1-SPACE.

A previous study reported that the signal intensity of plaques on T1-weighted MR imaging could classify plaques according to histopathologic types [7]. In the present study, the correlation of SR of plaques between T1-SPACE and CS-T1-SPACE was statistically significant, but we didn’t investigate CS-T1-SPCE accurately characterized intraplaque components. There may exist some overestimation of plaque size on CS-T1-SPACE because plaque area of CS-T1-SPACE was consistently larger than that of T1-SPACE although the correlation of plaque area between T1-SPACE and CS-T1-SPACE was very strong. However, we have demonstrated that CS-T1-SPACE is very useful for examining the existence of plaque, which would be beneficial for patients and physicians since CS-T1-SPACE may offer more opportunities for therapeutic interventions to prevent cerebral infarction.

In this study, T1-SPACE was accelerated by parallel imaging (PI). PI is now routinely used as a k-space undersampling method by reducing the number of phase-encoding steps. However, the acceleration factor for PI is usually no higher than 2 or 3 with non-contrast MRI, because a further reduction in phase-encoding steps will cause substantial increases in the noise associated with the geometry factor (g-factor) [26]. In contrast, CS is expected to achieve higher k-space undersampling by exploiting the underlying sparsity of the image in an appropriate transform domain [16], and some 
studies have shown CS could achieve better image quality compared with PI [10]. The CS-T1-SPACE sequence reduces acquisition time from $7 \min 9 \mathrm{~s}$ to $2 \min 49 \mathrm{~s}$ with the same resolution, and the acquisition time for CS-T1-SPACE is thus approximately 2.5-times shorter than that of T1-SPACE, which will be beneficial for patients. Patients who have carotid plaques tend to be older and some of these patients have difficulty staying still for extended periods, and reducing the acquisition time minimizes motion artifacts. Although CS reduces acquisition time, a long time is usually required to reconstruct images after scanning [11]. However, in this study, total reconstruction was finished within $2 \min 15 \mathrm{~s}$, and a shorter reconstruction time makes the CS-T1-SPACE more useful in clinical practice.

A few limitations to this study need to be considered. First, this study undertook no histological validation of plaques, and we could not histopathologically confirm the existence or characteristics of plaques nor the 7 plaques which were solely identified on CS-T1-SPACE were the lesions, but the purpose of this study was to compare visualization between T1-SPACE and CS-T1-SPACE. Second, we used a prototype-accelerated technique, which was developed for general application and had not been fully optimized for carotid vessel walls. Further optimization of CS technique could potentially improve the visualization of carotid plaque in CS-T1-SPACE. In addition to CS technique, there are several differences of imaging parameters between T1-SPACE and CS-T1-SPACE such as TR, FOV, acceleration factors. These differences may also affect image quality and the point spread function due to the difference in k-space acquisition ordering. Third, although the name of image sequence was blinded, we did not evaluate visualization score separately. Fourth, the reconstructed images should 
become constant after iterative reconstruction with regularization constraint, however, representative edge sharpness shown in Figure 2 showed sharpness slightly changes during iteration process even after 10 iteration times. This is partly because sharpness was calculated by division process and slight signal changes affect the results much, and another reason is CS-T1-SPACE used in this study was a prototype one. There may exist early stopping issue and the image quality difference between 10 and 35 or more iterations shown in Figure 1, further exploration of adapting the number of iterations for patients and the optimization of image sequence are expected to improve the image quality in the future. "Sharpness" was used in this study, however, image contrast between the lumen and plaque or vessel wall were alternate terms. The other limitation was Kappa values for carotid plaques and vessel walls showed good agreement in CS-T1-SPACE, but worse than T1-SPACE. It is presumed that image blurring affected scoring in CS-T1-SPACE. Nevertheless, better sharpness proved better visualization in CS-T1-SPACE.

In conclusion, CS-T1-SPACE revealed better visualization scores and sharpness in less than 2.5times the scan time compared with T1-SPACE in the evaluation of carotid plaques and vessel walls in the ICA. CS-T1-SPACE offers a promising method for investigating carotid vessel walls due to the better image quality with shorter acquisition time. 


\section{References}

1 Feigin VL, Forouzanfar MH, Krishnamurthi R et al (2014) Global and regional burden of stroke during 1990-2010: findings from the Global Burden of Disease Study 2010. Lancet $383: 245-254$

Altaf N, MacSweeney ST, Gladman J, Auer DP (2007) Carotid intraplaque hemorrhage predicts recurrent symptoms in patients with high-grade carotid stenosis. Stroke 38:1633-1635

Wityk RJ, Lehman D, Klag M, Coresh J, Ahn H, Litt B (1996) Race and sex differences in the distribution of cerebral atherosclerosis. Stroke 27:1974-1980

Vermeer SE, Den Heijer T, Koudstaal PJ et al (2003) Incidence and risk factors of silent brain infarcts in the population-based Rotterdam Scan Study. Stroke 34:392-396

5 Sun J, Zhao XQ, Balu N et al (2017) Carotid Plaque Lipid Content and Fibrous Cap Status Predict Systemic CV Outcomes: The MRI Substudy in AIM-HIGH. JACC Cardiovasc Imaging $10: 241-249$

6 Zhao XQ, Hatsukami TS, Hippe DS et al (2014) Clinical factors associated with high-risk carotid plaque features as assessed by magnetic resonance imaging in patients with established vascular disease (from the AIM-HIGH Study). Am J Cardiol 114:1412-1419

7 Narumi S, Sasaki M, Natori T et al (2015) Carotid plaque characterization using 3D T1weighted MR imaging with histopathologic validation: a comparison with 2D technique. AJNR Am J Neuroradiol 36:751-756 
Zhang L, Zhang N, Wu J et al (2015) High resolution three dimensional intracranial arterial wall imaging at 3 T using T1 weighted SPACE. Magn Reson Imaging 33:1026-1034

Boussel L, Herigault G, de la Vega A, Nonent M, Douek PC, Serfaty JM (2006) Swallowing, arterial pulsation, and breathing induce motion artifacts in carotid artery MRI. J Magn Reson Imaging 23:413-415

Yamamoto T, Fujimoto K, Okada T et al (2016) Time-of-Flight Magnetic Resonance Angiography With Sparse Undersampling and Iterative Reconstruction: Comparison With Conventional Parallel Imaging for Accelerated Imaging. Invest Radiol 51:372-378

11 Fushimi Y, Fujimoto K, Okada T et al (2016) Compressed Sensing 3-Dimensional Time-ofFlight Magnetic Resonance Angiography for Cerebral Aneurysms: Optimization and Evaluation. Invest Radiol 51:228-235

Stalder AF, Schmidt M, Quick HH et al (2015) Highly undersampled contrast-enhanced MRA with iterative reconstruction: Integration in a clinical setting. Magn Reson Med 74:1652-1660

13 Rossi Espagnet MC, Bangiyev L, Haber M et al (2015) High-Resolution DCE-MRI of the Pituitary Gland Using Radial k-Space Acquisition with Compressed Sensing Reconstruction. AJNR Am J Neuroradiol 36:1444-1449

14 Fritz J, Raithel E, Thawait GK, Gilson W, Papp DF (2016) Six-Fold Acceleration of HighSpatial Resolution 3D SPACE MRI of the Knee Through Incoherent k-Space Undersampling and Iterative Reconstruction-First Experience. Invest Radiol 51:400-409 
15 Altahawi FF, Blount KJ, Morley NP, Raithel E, Omar IM (2017) Comparing an accelerated 3D fast spin-echo sequence (CS-SPACE) for knee 3-T magnetic resonance imaging with traditional 3D fast spin-echo (SPACE) and routine 2D sequences. Skeletal Radiol 46:7-15

16 Lustig M, Donoho D, Pauly JM (2007) Sparse MRI: The application of compressed sensing for rapid MR imaging. Magn Reson Med 58:1182-1195

17 Yuan J, Usman A, Reid SA et al (2017) Three-dimensional black-blood T2 mapping with compressed sensing and data-driven parallel imaging in the carotid artery. Magn Reson Imaging 37:62-69

Yuan J, Usman A, Reid SA et al (2017) Three-dimensional black-blood multi-contrast carotid imaging using compressed sensing: a repeatability study. Magma. 10.1007/s10334-017-06401

19 Li B, Li H, Kong H, Dong L, Zhang J, Fang J (2017) Compressed sensing based simultaneous black- and gray-blood carotid vessel wall MR imaging. Magn Reson Imaging 38:214-223

20 Makhijani MK, Balu N, Yamada K, Yuan C, Nayak KS (2012) Accelerated 3D MERGE carotid imaging using compressed sensing with a hidden Markov tree model. J Magn Reson Imaging 36:1194-1202

21 Li G, Zaitsev M, Buchert M et al (2015) Improving the robustness of 3D turbo spin echo imaging to involuntary motion. Magma 28:329-345 
arbitrary k-space trajectories. Magn Reson Med 46:638-651

23 Liang D, Liu B, Wang J, Ying L (2009) Accelerating SENSE using compressed sensing. Magn

Reson Med 62:1574-1584

24 Boubertakh R, Prieto C, Batchelor PG et al (2009) Whole-heart imaging using undersampled radial phase encoding (RPE) and iterative sensitivity encoding (SENSE) reconstruction. Magn Reson Med 62:1331-1337

25 Kramer JH, Arnoldi E, François CJ et al (2013) Dynamic and static magnetic resonance angiography of the supra-aortic vessels at $3.0 \mathrm{~T}$ : intraindividual comparison of gadobutrol, gadobenate dimeglumine, and gadoterate meglumine at equimolar dose. Invest Radiol 48:121128

26 Griswold MA, Jakob PM, Heidemann RM et al (2002) Generalized autocalibrating partially parallel acquisitions (GRAPPA). Magn Reson Med 47:1202-1210 


\section{Figure Legends}

\section{Figure 1}

A 75-year-old man with left ICA plaque. Sagittally reconstructed images of CS-T1-SPACE with variable iterations (1, 2, 3, 4, 5, 10, 15, 20, 25, 30, 35, 40, 45 and 50). High-intensity plaques are shown at the left ICA, and appear better demarcated according to the number of iterations (arrows). Multiple aliasing artifacts are shown in figures with fewer iterations, but these become invisible with more iterations (arrowheads). Note that the number shown in the left lower corner of each image represents the number of iterations. T1-SPACE image is also shown $\left(^{*}\right)$.

\section{Figure 2}

Edge sharpness at the edge of the carotid artery for CS-T1-SPACE images with each iteration of Figure 1. Sharpness was measured on 4 proximal and distal part of bilateral ICAs. Sharpness is defined as the slope between $20 \%$ and $80 \%$ of maximum signal intensity for the Gaussian-fitted line profile of the carotid artery wall and the carotid lumen as well as the neighboring structure on the source image. Sharpness rapidly increases from 1 to 10 iterations, then slightly fluctuated between 10 and 50 iterations. Sharpness of T1-SPACE was also shown at the iteration time of "0". Sharpness of CS-T1SPACE with 10 iteration times are larger than that of T1-SPACE

Figure 3 
An 84-year-old man with right ICA plaque imaged using T1-SPACE (a, oblique coronal plane; c, axial plane) and CS-T1-SPACE (b, oblique coronal plane; d, axial plane). White arrows indicate common carotid artery and ICA. Arrowheads indicate carotid plaque. Scores for visualization of the carotid plaque and vessel wall on T1-SPACE and CS-T1-SPACE are all 5. The plaque and vessel wall are equally well visualized on CS-T1-SPACE and T1-SPACE.

\section{Figure 4.}

An 83-year-old man without carotid plaque (a, T1-SPACE; b, CS-T1-SPACE). White arrows indicate common carotid artery and ICA. Scores for visualization of the vessel wall on T1-SPACE and CS-T1-SPACE are all 5. The vessel walls are equally well visualized on CS-T1-SPACE and T1SPACE. Note that CS technique and several differences of imaging parameters between CS-T1SPACE and T1-SPACE may cause slightly different appearance of vessel wall between them.

\section{Figure 5.}

An 83-year-old man with left ICA plaque (a, left carotid artery on T1-SPACE; b, left carotid artery on CS-T1-SPACE; c, right carotid artery on T1-SPACE; d, right carotid artery CS-T1-SPACE). White arrows indicate common carotid artery and ICA. Motion artifacts associated swallowing is seen. Arrowheads indicate carotid plaque. Scores for the plaque and vessel on T1-SPACE are both 1, and scores on CS-T1-SPACE are both 3. T1-SPACE images are affected by motion artifacts. 


\section{Figure 6.}

Scores for quality of visualization for carotid plaque (a) and vessel wall (b). Scores are significantly higher for CS-T1-SPACE than for T1-SPACE.

\section{Supplementary Figure 1}

Inclusion and exclusion criteria of plaques are shown.

\section{Supplementary Figure 2}

Scatter plots of SR in plaque between T1-SPACE and CS-T1-SPACE. The correlation is significant.

\section{Supplementary Figure 3}

Scatter plots of plaque area size between T1-SPACE and CS-T1-SPACE.

\section{Supplementary Figure 4}

Bland-Altman plot of the plaque size on T1-SPACE and CS-T1-SPACE showed most of plots are shown between +/- 1.96 SDs. 


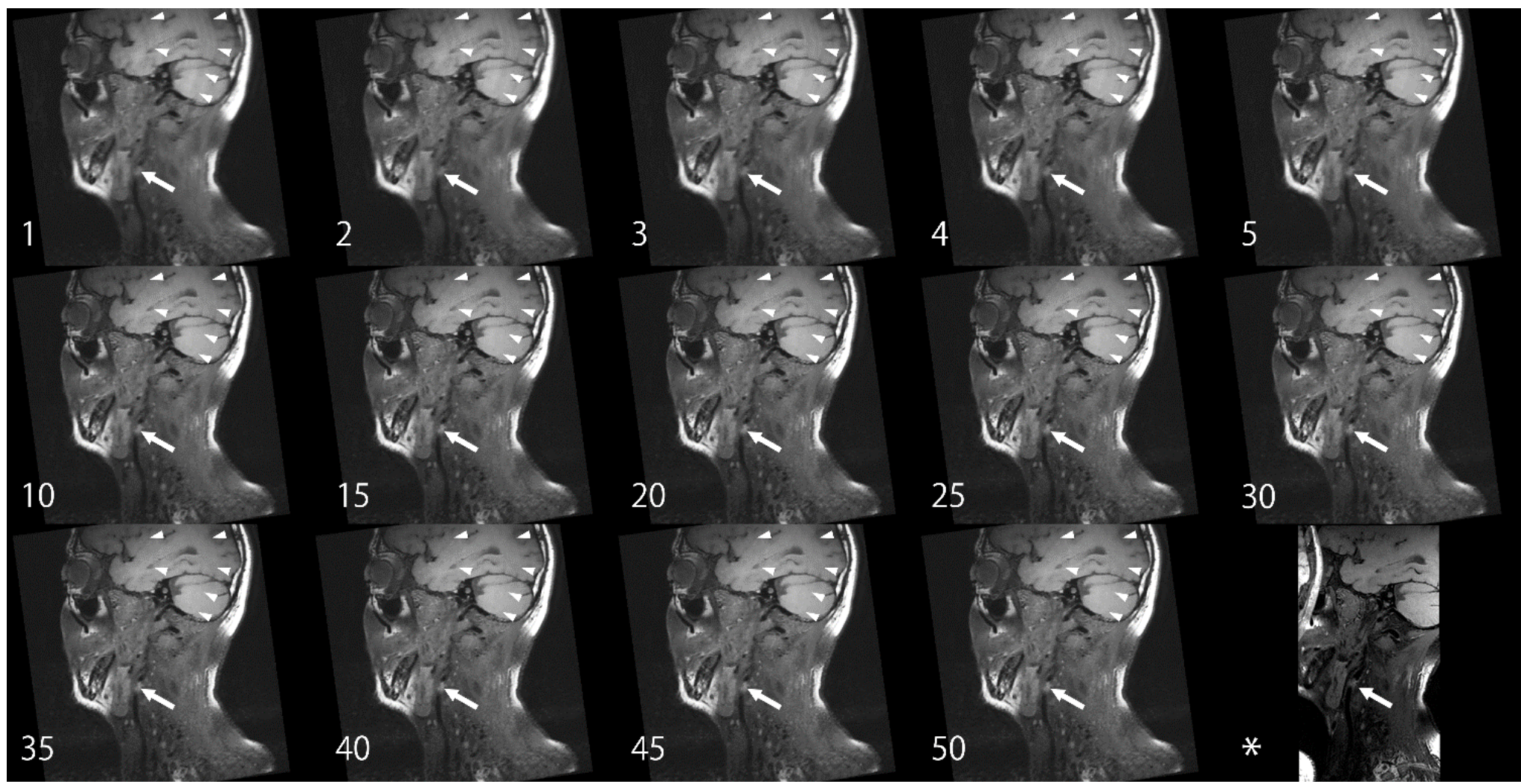


Figure 2

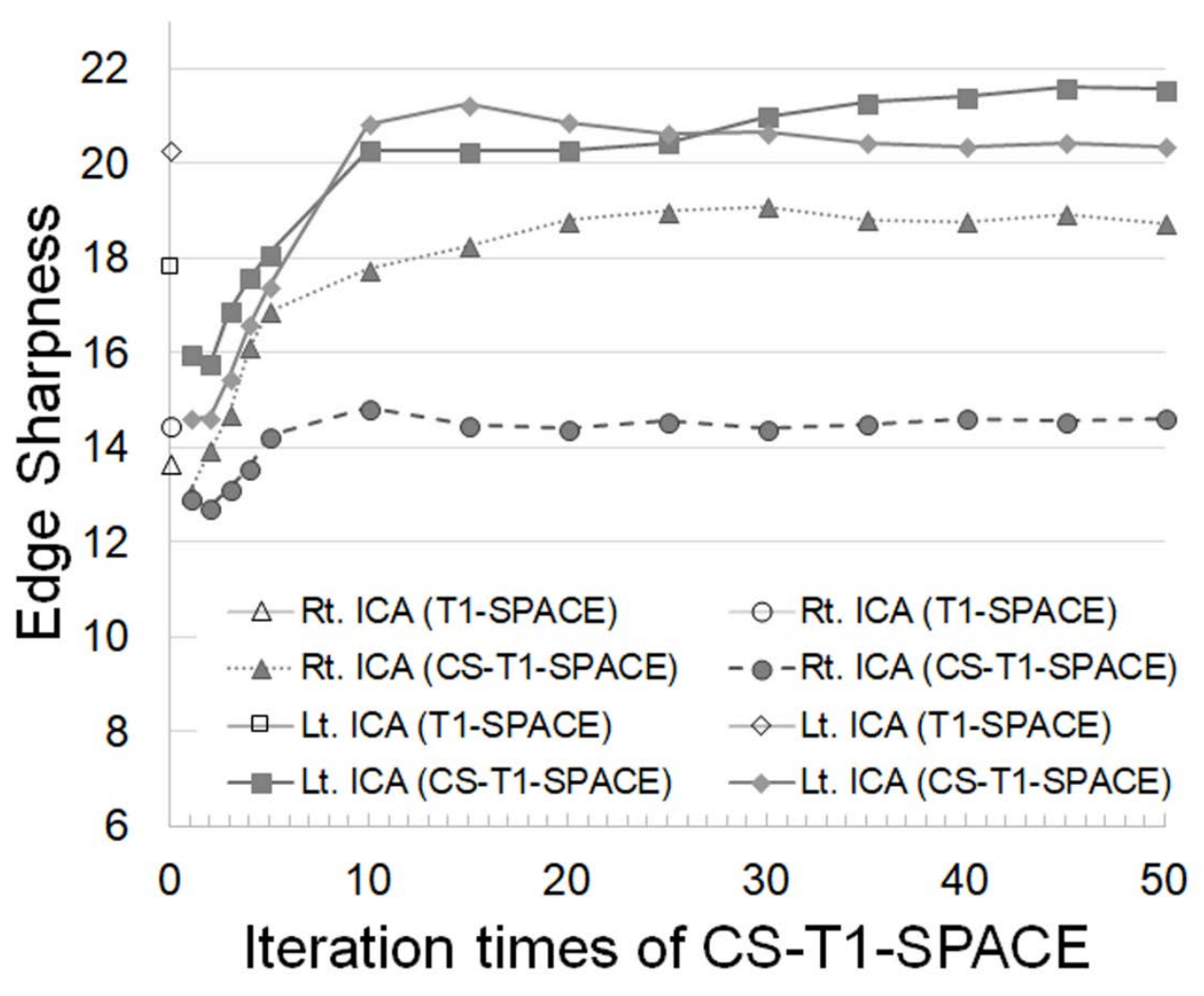


Figure 3

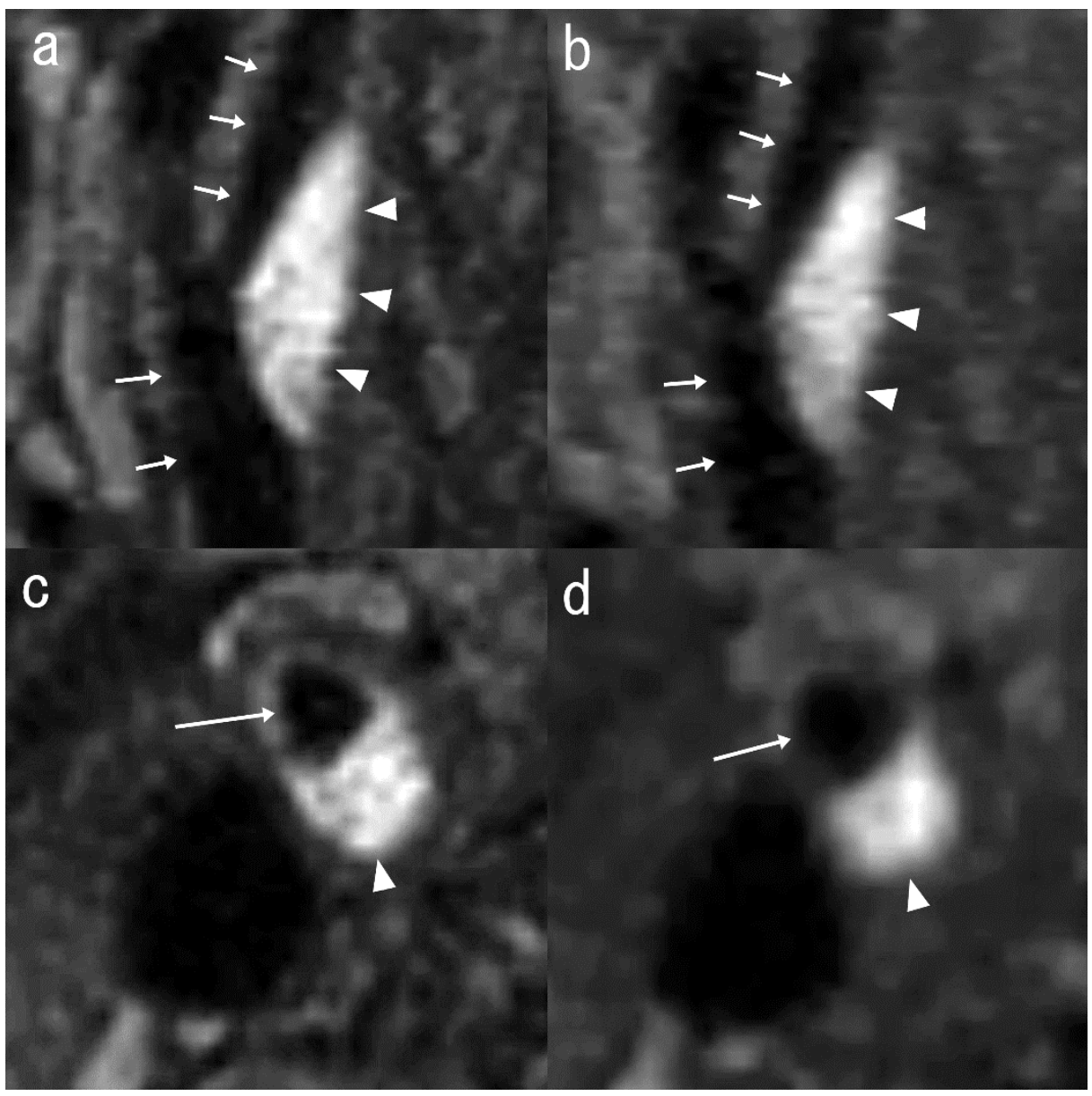


Figure 4.

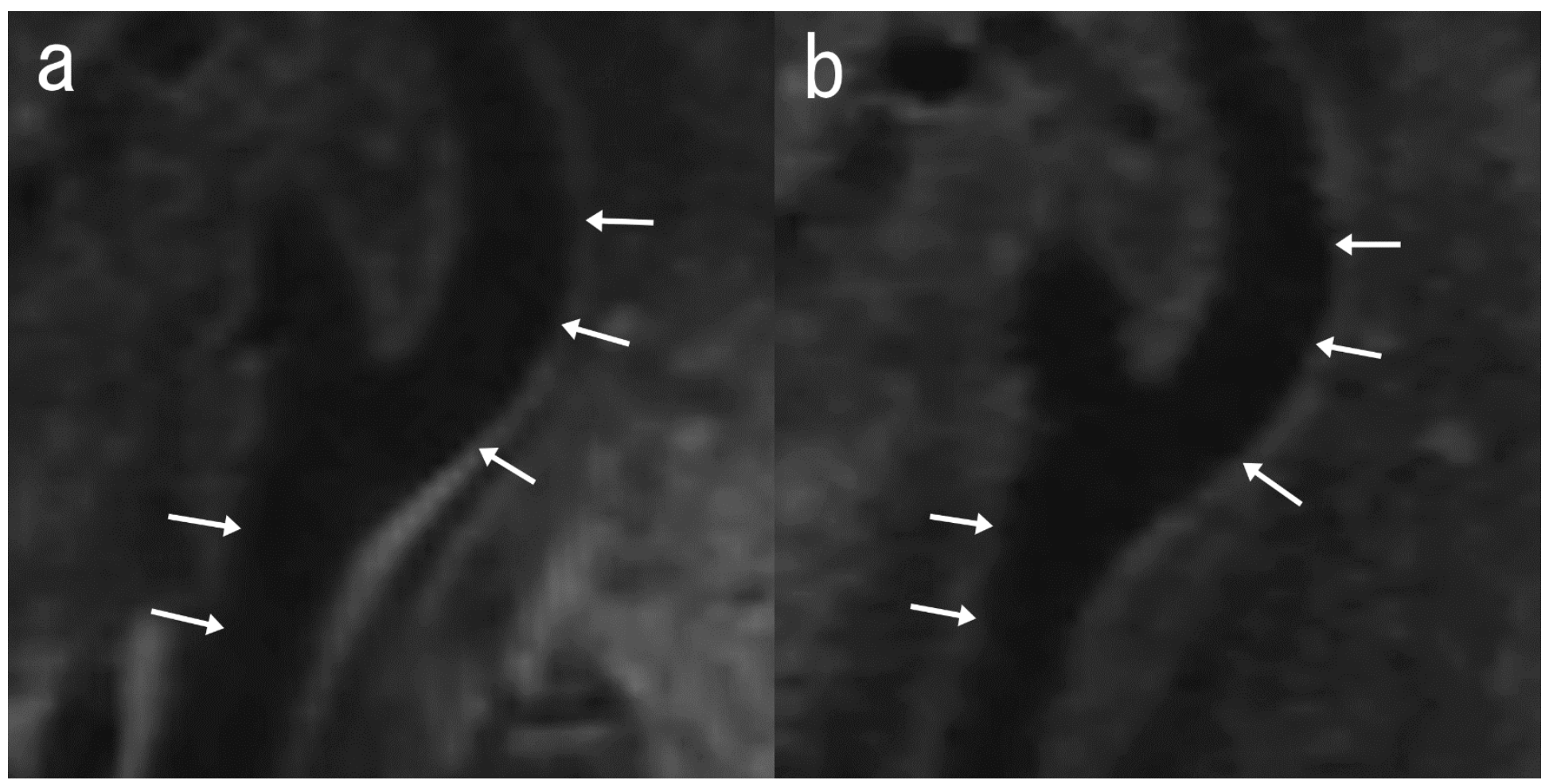


Figure 5.

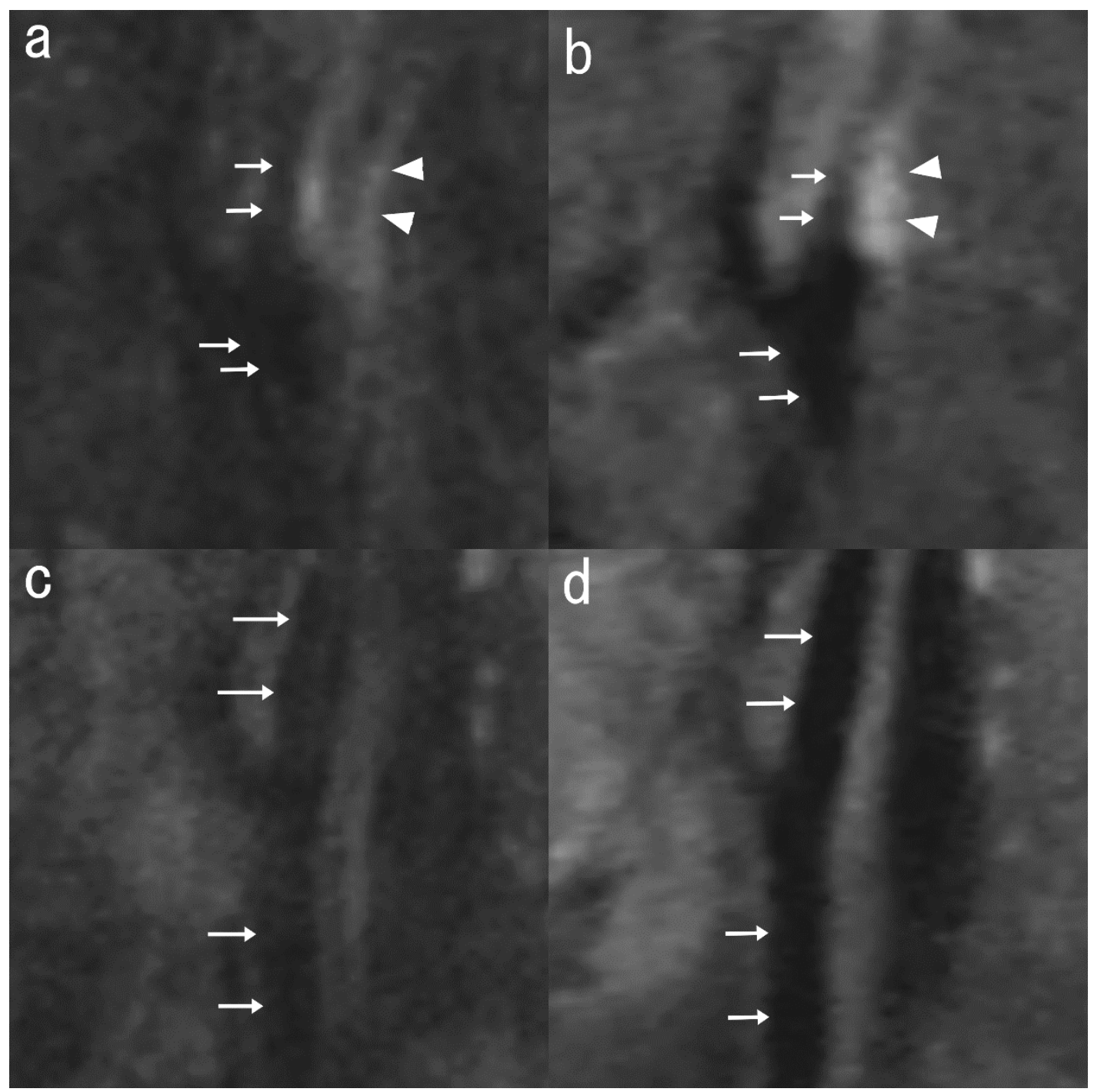


Figure 6.

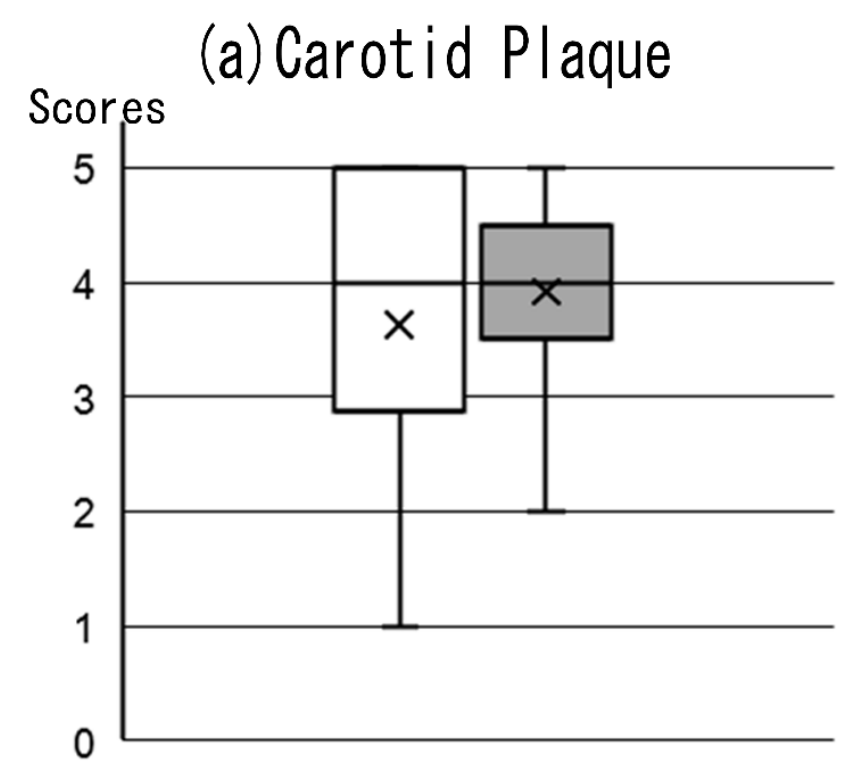

$\square$ T1-SPACE $\square$ CS-T1-SPACE

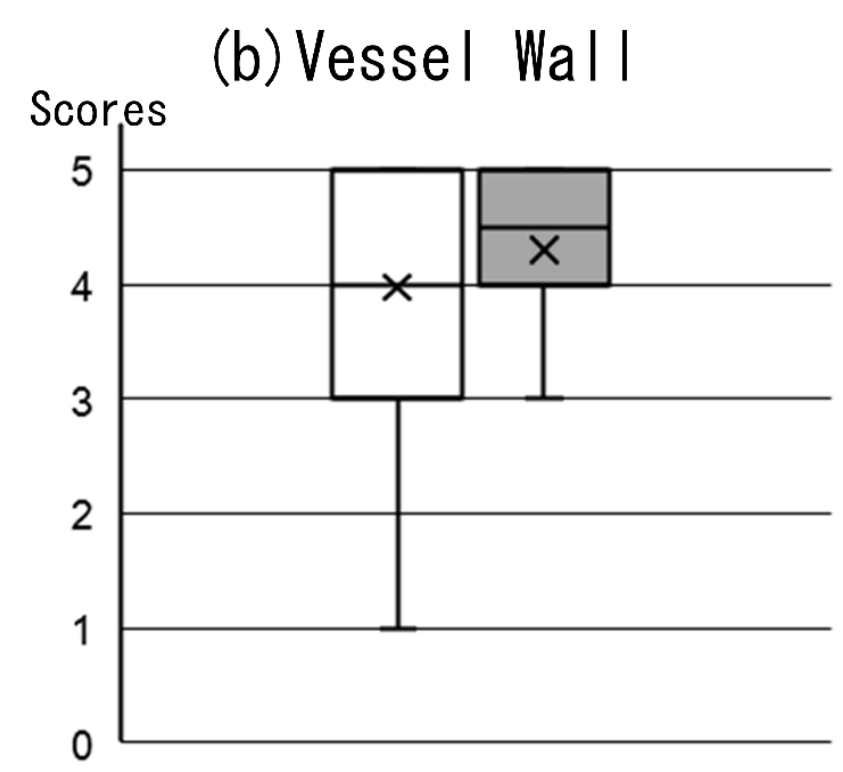

$\square$ T1-SPACE $\square$ CS-T1-SPACE 
Supplementary Figure 1

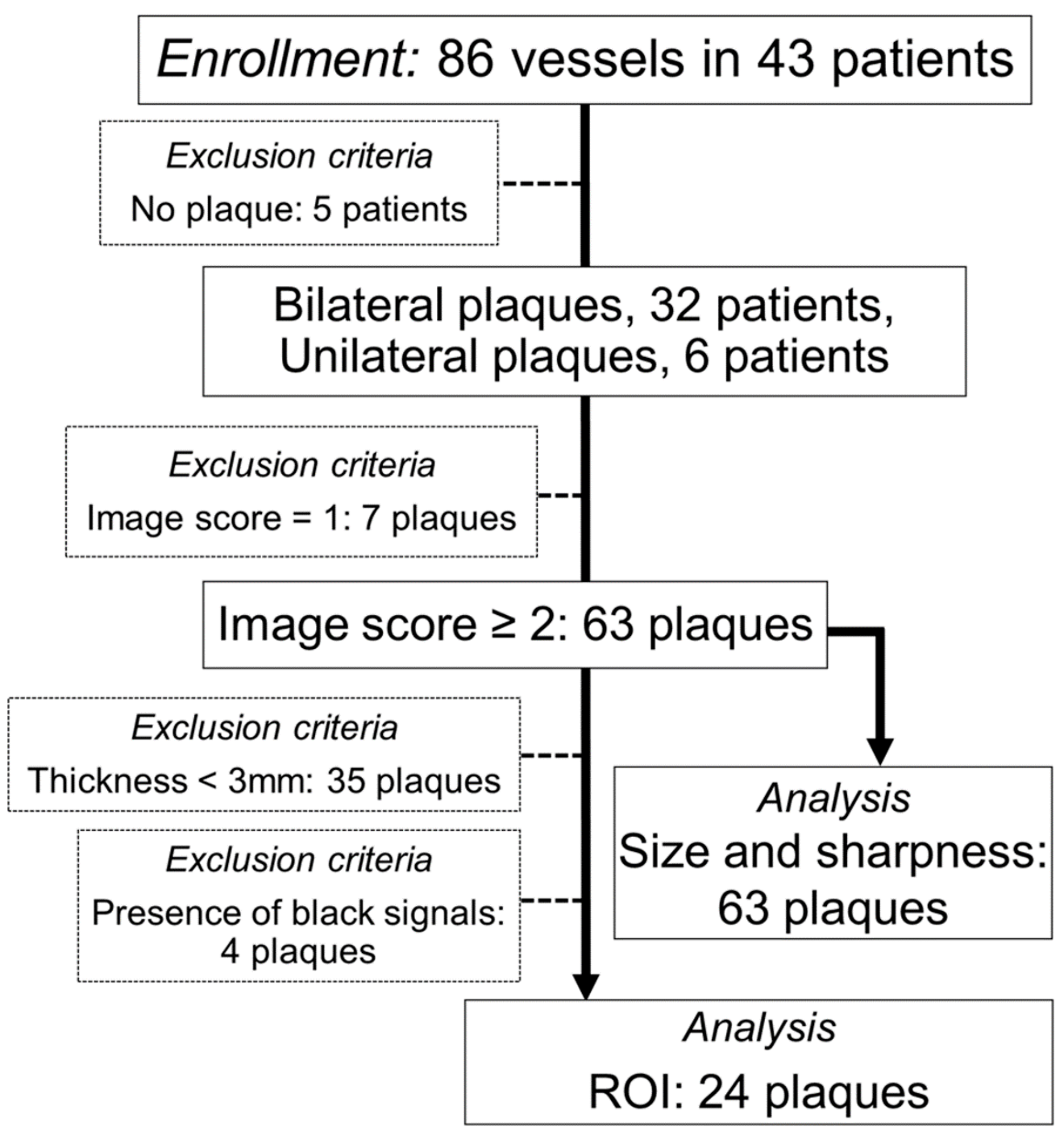


Supplementary Figure 2

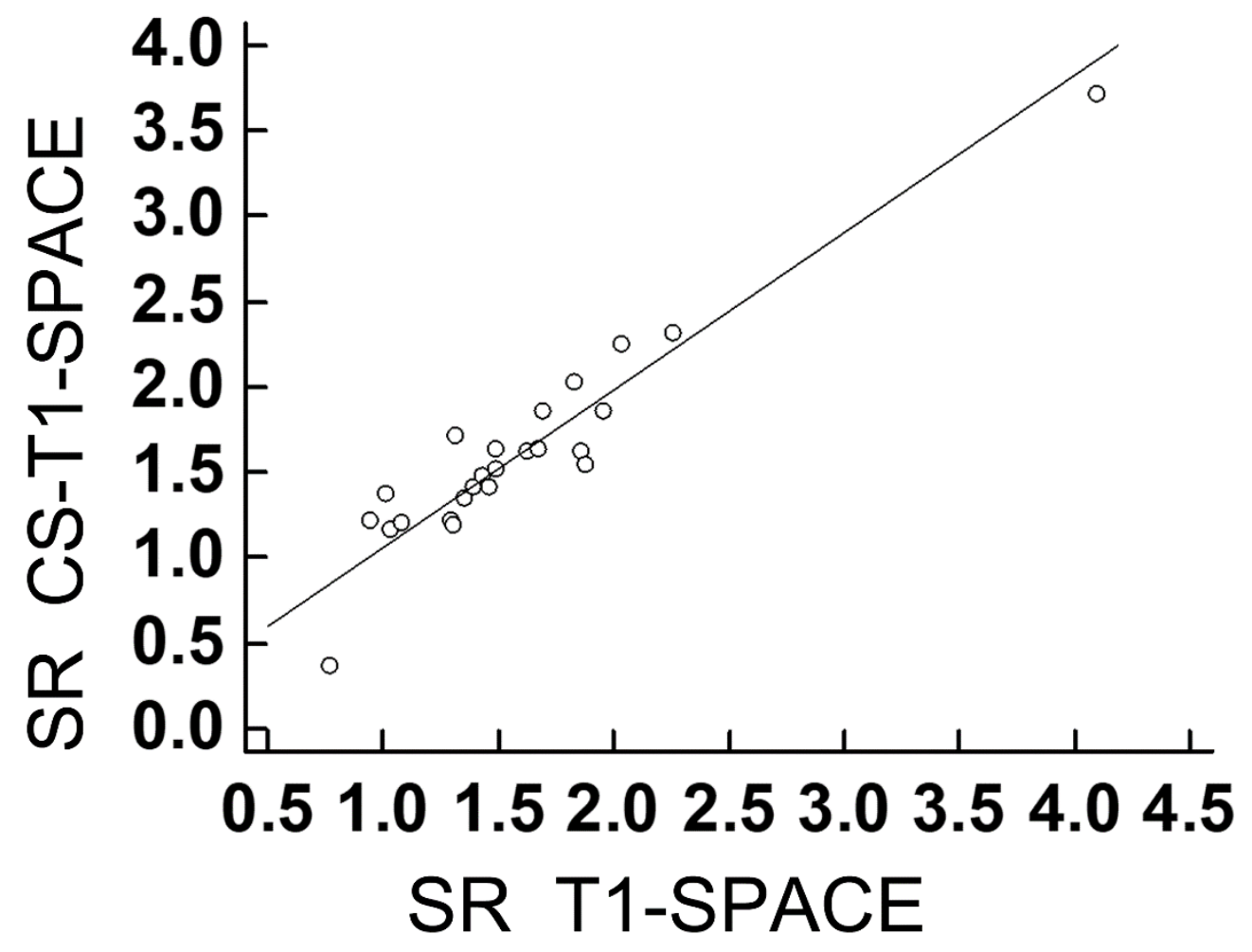


Supplementary Figure 3

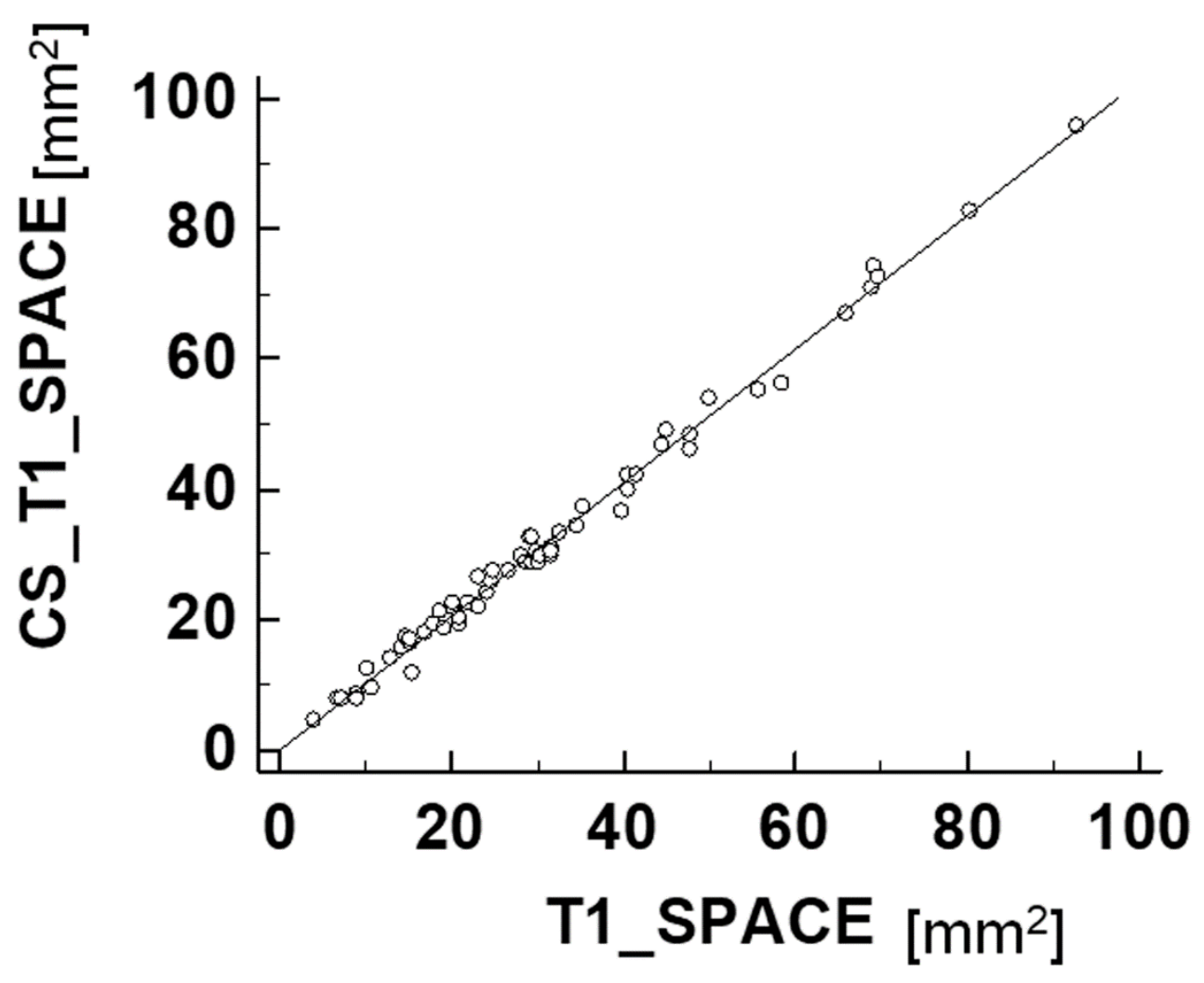


Supplementary Figure 4

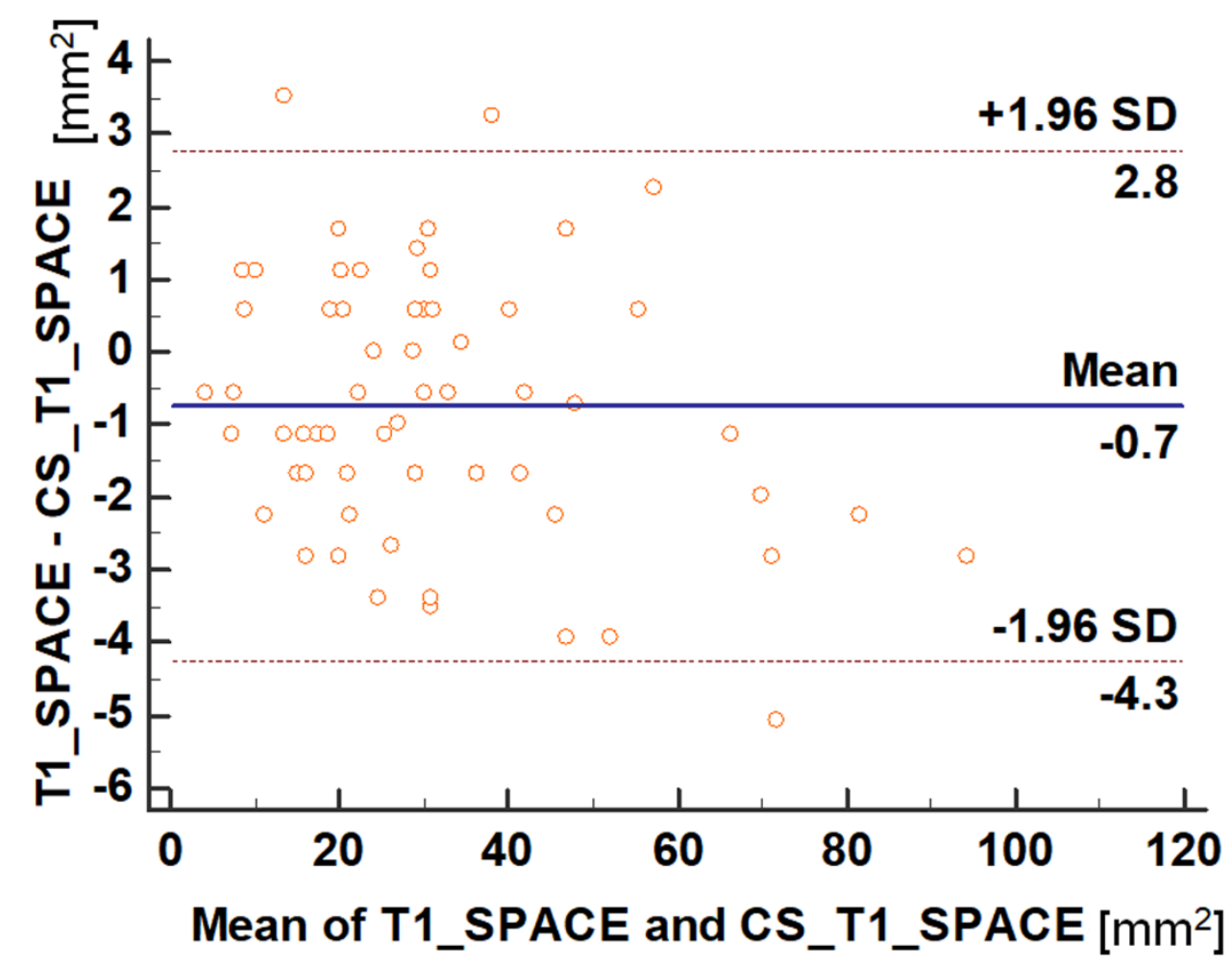

